\title{
Determination of Chiral Perturbarion Theory low energy constants from a precise description of $\pi \pi$ scattering threshold parameters
}

\author{
Guillermo Rios* \\ Universidad de Murcia \\ E-mail: g.rios.marquez@um.es \\ Jenifer Nebreda \\ Yukawa Institute for Theoretical Physics, Kyoto University \\ E-mail: jnebreda@yukawa.kyoto-u.ac.jp \\ Jose R. Pelaez \\ Universidad Complutense de Madrid \\ E-mail: jrpelaezafis.ucm.es
}

\begin{abstract}
We report on our determination of the values of the one and two loop low energy constants appearing in the Chiral Perturbation Theory calculation of the $\pi \pi$ scattering amplitude. For this we use a precise sum rule determination of scattering lengths and slopes that appear in the effective range expansion. In addition we provide new sum rules and the values for these coefficients up to third order in the expansion. Our results when using only the scattering lengths and slopes of the S, P, D and F waves are consistent with previous determinations, but seem to require higher order contributions if they are to accommodate the third order coefficients of the effective range expansion.
\end{abstract}

The 7th International Workshop on Chiral Dynamics,

August 6-10, 2012

Jefferson Lab, Newport News, Virginia, USA

\footnotetext{
* Speaker.
} 


\section{Introduction}

Chiral Perturbation Theory (ChPT) [1,2] describes the low energy interactions of pions, which are the pseudo-Goldstone bosons associated to the spontaneus chiral symmetry breaking of QCD, and hence, the relevant degrees of freedom at low energies. ChPT is built as the most general low energy expansion in terms of the pion momenta and mass that is compatible with the QCD symmetries. The details of the underlying dynamics at higher energies are encoded in a set of parameters, known as low energy constants (LECs), that appear at the different orders in the expansion, and, once renormalized, absorb the loop divergences present at that order. Since perturbative QCD cannot be applied at very low energies, it is particularly difficult to obtain the values of these LECs from first principles and, with few exceptions, the LECs have been determined best from the comparison with experiment $[2,3,4,5]$. There are determinations of the LECs from lattice QCD (we refer to [7] for a recent compilation), and it is also possible to obtain positivity constraints for the LECs coming from axiomatic field theory [6].

In the ChPT $\pi \pi$ scattering amplitude only certain combiantions of the LECs appear to a given order of the calculation. To leading order, $O\left(p^{2}\right)$, there are no LECs and only the pion mass decay constant $M_{\pi}$ and $f_{\pi}$ appear. To next to leading order (NLO), or $O\left(p^{4}\right)$, which corresponds to a oneloop calculation, only four LECs, called $l_{1}, \ldots, l_{4}$ appear in the amplitude. At next to next to leading order (NNLO), or $O\left(p^{6}\right)$, which corresponds to a two-loop calculation, only six independent terms appear [8], multiplied the corresponding combination of LECs, denoted $\bar{b}_{i}, i=1 \ldots 6$.

Here we report our work [9] on the determination of the LECs that appear in the $\pi \pi$ scattering amplitude. We obtain these LECs from fits to the coefficients of the momentum expansion of the amplitude around threshold, known as threshold parameters, which can be calculated within ChPT and expressed in terms of the LECs. The experimental value of the threshold parameters we use is the one obtained from a dispersive data analysis in [10], which includes the very precise and reliable results on $K_{l 4}$ decays from the NA48/2 collaboration [12]. We also provide here the calculation of the third order coefficients of the threshold expansion, which was not performed in [10].

\section{Threshold parameters}

The amplitude for $\pi \pi$ scattering is customarily decomposed in terms of partial waves $t_{\ell}^{I}$, of definite isospin $I$ and angular momentum $\ell: t_{\ell}^{I}(s)=\frac{1}{64 \pi} \int_{-1}^{1} T^{I}(s, t, u) P_{\ell}(\cos \theta) d(\cos \theta), \theta$ being the scattering angle, $P_{\ell}$ the Legendre polynomials, $s, t, u$ the usual Mandelstam variables and $T$ the amplitude. With this normalization, the threshold expansion can be written as:

$$
\frac{1}{M_{\pi}} \operatorname{Re} t_{\ell}^{I}(s)=p^{2 \ell}\left(a_{\ell I}+b_{\ell I} p^{2}+\frac{1}{2} c_{\ell I} p^{4}+\ldots\right),
$$

where the $a_{\ell I}$ are usually called scattering lengths, the $b_{\ell I}$ slope parameters, the $c_{\ell I}$ shape parameters, and all of them, generically, threshold parameters.

The use of sum rules to obtain the values of threshold parameters is a well established technique $[5,11]$ that we will also use here. Our experimental determination of the threshold parameters is performed using the parametrizations of [10], which were obtained by highly constraining data fits to satisfy three sets of dispersion relations within uncertainties. In [10], the values of the $a$ and $b$ parameters up to $\mathrm{F}$ waves were provided. With the aim of minimizing the uncertainties, they 
were obtained from sum rules, with the only exception of the $5 a_{S 0}+2 a_{S 2}$ combination, which is orthogonal to the one appearing in the Olsson sum rule (note the spectroscopic notation, where the $\ell=0,1,2,3 \ldots$ are denoted S,P,D,F...).

Here, we also provide the calculation of the third order coefficients $c$ of the threshold expansion, which adds five more observables for the fit. For the $c$ parameters with $\ell>0$, we use the Froissart-Gribov sum rules:

$$
\begin{aligned}
c_{\ell I}=\frac{\sqrt{\pi} \Gamma(\ell+1)}{M_{\pi} \Gamma(\ell+3 / 2)} \int_{4 M_{\pi}^{2}}^{\infty} d s\{ & \frac{16 \operatorname{Im} F^{I^{\prime \prime}}\left(s, 4 M_{\pi}^{2}\right)}{\left(s-4 M_{\pi}^{2}\right)^{2} s^{\ell+1}} \\
& \left.-8(\ell+1) \frac{\operatorname{Im} F^{I^{\prime}}\left(s, 4 M_{\pi}^{2}\right)}{\left(s-4 M_{\pi}^{2}\right) s^{\ell+2}}+\frac{\operatorname{Im} F^{I}\left(s, 4 M_{\pi}^{2}\right)}{s^{\ell+3}} \frac{(\ell+2)^{2}(\ell+1)}{\ell+3 / 2}\right\},
\end{aligned}
$$

where $F^{I}(s, t)=T^{I}(s, t) / 4 \pi^{2}$ and the primes denote the derivative with respect to $\cos \theta$. This formula allow us to calculate the $c$ parameters for the P, D0, D2 and F waves. For the S waves we provide two new sum rules, and also one for $c_{P}$, in order to reduce its error:

$$
\begin{aligned}
& c_{S 2}=-6 b_{P}-10 a_{D 2}+\frac{8}{M_{\pi}} \int_{4 M_{\pi}^{2}}^{\infty} d s\left\{\frac{\operatorname{Im} F^{0+}(s, 0)}{s^{3}}\right. \\
&+\left.\frac{1}{\left(s-4 M_{\pi}^{2}\right)^{5 / 2}}\left[\frac{\operatorname{Im} F^{0+}(s, 0)}{\sqrt{s-4 M_{\pi}^{2}}}-\frac{2 M_{\pi} a_{S 2}^{2}}{\pi}-\frac{s-4 M_{\pi}^{2}}{\pi}\left(\frac{M_{\pi}}{2}\left(2 a_{S 2} b_{S 2}+a_{S 2}^{4}\right)-\frac{a_{S 2}^{2}}{4 M_{\pi}}\right)\right]\right\} \\
& c_{S 0}=-2 c_{S 2}-20 a_{D 2}-10 a_{D 0}+\frac{12}{M_{\pi}} \int_{4 M_{\pi}^{2}}^{\infty} d s\left\{\frac{\operatorname{Im} F^{00}(s, 0)}{s^{3}}+\frac{1}{\left(s-4 M_{\pi}^{2}\right)^{5 / 2}}\left[\frac{\operatorname{Im} F^{00}(s, 0)}{\sqrt{s-4 M_{\pi}^{2}}}\right.\right. \\
&\left.\left.-\frac{4 M_{\pi}\left(2 a_{S 2}^{2}+a_{S 0}^{2}\right)}{3 \pi}-\frac{s-4 M_{\pi}^{2}}{3 \pi}\left(M_{\pi}\left[2\left(2 a_{S 2} b_{S 2}+a_{S 2}^{4}\right)+2 a_{S 0} b_{S 0}+a_{S 0}^{4}\right]-\frac{2 a_{S 2}^{2}+a_{S 0}^{2}}{2 M_{\pi}}\right)\right]\right\} \\
& c_{P}=-\frac{14 a_{F}}{3}+\frac{16}{3 M_{\pi}} \int_{4 M_{\pi}^{2}}^{\infty} d s\left\{\frac{\operatorname{Im} F^{I=0}(s, 0)}{3 s^{4}-\frac{\operatorname{Im} F^{I=1}(s, 0)}{2 s^{4}}}\right. \\
&\left.\quad-\frac{5 \operatorname{Im} F^{I=2}(s, 0)}{6 s^{4}}+\left[\frac{\operatorname{Im} F^{I=1}(s, 0)}{\left(s-4 M_{\pi}^{2}\right)^{4}}-\frac{3 a_{P}^{2} M_{\pi}}{4 \pi\left(s-4 M_{\pi}^{2}\right)^{3 / 2}}\right]\right\} .
\end{aligned}
$$

The derivation is similar to that of the sum rules for $b_{P}, b_{S 0}$ and $b_{S 2}$ obtained in [13]. They correspond to the threshold limit, taken from above, of the second derivative of a forward dispersion relation for the $F^{I=1}, F^{0+}$ and $F^{00}$ amplitudes, respectively. Let us recall that $F^{0+}=F^{I=2} / 2+F^{I=1} / 2$ whereas $F^{00}=2 F^{I=2} / 3+F^{I=0} / 3$. For a list of the resulting values, we refer the reader to Table II of our original work [9].

\section{3. $O\left(p^{4}\right)$ fits}

We start by fitting the $O\left(p^{4}\right)$ LECs by using the one-loop expression of the threshold parameters, which will help us check the stability of the LECs values and the need for higher orders. We actually fit the $\bar{l}_{i}$ parameters, which are basically the $l_{i}^{r}(\mu)$ at the $\mu=M_{\pi}$ scale and normalized so that they have values of order one [2]. Note, however, that $\bar{l}_{3}$ and $\bar{l}_{4}$ only appear through the quark 
Determination of ChPT LECs from a precise description of $\pi \pi$ scattering threshold parameters Guillermo Rios

\begin{tabular}{lccccc}
\hline \hline Fit to & $\bar{l}_{1}$ & $\bar{l}_{2}$ & $\bar{l}_{3}$ & $\bar{l}_{4}$ & $\chi^{2} /$ d.o.f. \\
\hline$a_{S}, b_{S}, a_{P}$ & $1.1 \pm 1.0$ & $5.1 \pm 0.7$ & $-1 \pm 8$ & $7.1 \pm 0.7$ & 0.23 \\
$a_{D}$ & $-1.75 \pm 0.22$ & $5.91 \pm 0.10$ & - & - & 0 \\
$c_{S}$ & $-2.4 \pm 0.9$ & $4.8 \pm 0.4$ & - & - & 0 \\
$a_{S}, b_{S}, a_{P}, a_{D}, c_{S}, b_{P}$ & $-2.06 \pm 0.14$ & $5.97 \pm 0.07$ & $-5 \pm 8$ & $7.1 \pm 0.6$ & 7.9 \\
$a_{S}, b_{S}, a_{P}, a_{D}, c_{S}, b_{P}$, using $f_{0}$ & $-1.06 \pm 0.11$ & $4.6 \pm 0.9$ & $0 \pm 6$ & $5.0 \pm 0.3$ & 7.06 \\
\hline Estimate $O\left(p^{4}\right)$ & $-1.5 \pm 0.5$ & $5.3 \pm 0.7$ & $-3 \pm 7$ & $6.0 \pm 1.2$ & - \\
\hline
\end{tabular}

Table 1: $O\left(p^{4}\right)$ fits to different sets of threshold parameters containing polynomial $O\left(p^{4}\right)$ contributions. We observe that a precise description of the observables is not possible at one loop. Anyway, we provide an estimate of how much one should enlarge the uncertainties of the LECs if, for simplicity, one still insists in using the one-loop formalism.

mass dependence of $M_{\pi}$ and $f_{\pi}$, respectively, and therefore we cannot expect much sensitivity to these two parameters from fits to the coefficients of the momentum expansion of amplitudes. In addition, since the LECs only appear in the polynomial part of the partial waves, which at one loop is of $O\left(p^{4}\right)$, only ten observables carry any dependence on the LECs: $a_{S 0)}, a_{S 2)}, a_{P}, b_{S 0}, b_{S 2}, b_{P}$, $c_{S 0}, c_{S 2}, a_{D 0}$ and $a_{D 2}$. The rest of the coefficients multiply powers of the momentum higher than $p^{4}$ and thus, do not receive a contribution from the $O\left(p^{4}\right)$ LECs.

In Table 1 we show the results of our fits. First, we have fitted only the observables whose leading contribution is of $O\left(p^{2}\right)$, since these might be more stable under the higher order corrections. The fit comes out with relatively low $\chi^{2} /$ d.o.f.. Next we present two determinations of $\bar{l}_{1}$ and $\bar{l}_{2}$, which can be fixed using only either $a_{D 0}$ and $a_{D 2}$, or $c_{S 0}$ and $c_{S 2}$. It is evident that the resulting values from those fits are incompatible. The incompatibility is even worse when fitting simultaneously the ten observables that depend on $\bar{l}_{i}$ to $O\left(p^{4}\right)$, where we obtain a high $\chi^{2} /$ d.o.f. value. Finally, the effect of higher order corrections has been studied by fitting to the one-loop amplitude but replacing $f_{\pi}$ by $f_{0}$ in the $O\left(p^{4}\right)$ terms, since the two expressions only differ in higher order contributions. This we show in row 5 of Table 1 . The $\chi^{2} /$ d.o.f is somewhat lower, but the values of the LECs come out rather different from the previous calculation.

These results imply that, to the present level of precision, the one-loop ChPT formalism is not enough and calls for higher order corrections. If one still wants to use this simpler version instead of the full two-loop amplitude one can include the effect of higher orders into a systematic uncertainty of the LECs. We propose to take the weighted average of the two previous fits, including a systematic uncertainty to cover the LECs values of both fits. In Table IV of the original reference [9], we compare the resulting threshold parameters obtained using this averaged set with the experimental values. Thanks to the larger uncertainty, the threshold parameters obtained are compatible within errors with the experimental values, except for $b_{S 0}$ and $b_{P}$, which differ by more than three and two standard deviations respectively. It is worth noting also that the positivity constranits obtained from firts principles [6] are perfectly satisfied by this set of LECs, even in the worst case scenario. 


\section{4. $O\left(p^{6}\right)$ fits}

As commented in the introduction, the two-loop $\pi \pi$ scattering amplitude can be recast in terms of six independent terms multiplied by their corresponding low energy constants $\bar{b}_{i}$. In turn, these $\bar{b}_{i}$ can be rewritten in terms of the four $O\left(p^{4}\right)$ LECs and six combinations $r_{i}$ of $O\left(p^{6}\right)$ LECs [14]. The difference in the amplitude using one way or the other is $O\left(p^{8}\right)$. However, despite increasing the number of parameters to ten, the $O\left(p^{6}\right)$ amplitude still provides just six independent structures. As a consequence, the fits in terms of $\bar{l}_{i}$ and $r_{i}$ are much more unstable, and can even lead to spurious solutions. For this reason we focus on the fits in terms of $\bar{b}_{i}$, and refer to the appendix [9] for a study of the $\bar{l}_{i}, r_{i}$ fits.

We first fit the ten threshold parameters used in the previous section because, having a non-zero $O\left(p^{4}\right)$ polynomial contribution, we expect these to be more stable under higher order corrections. In the first row of Table 2 we show the resulting $\bar{b}_{i}$, which describes fairly well the fitted observables with a $\chi^{2} /$ d.o.f. $=1.2$. However, when fitting all 18 observables, we obtain somewhat different LECs (see the second row of Table 2) and the $\chi^{2} /$ d.o.f. comes out rather poor. We have noticed that $c_{P}$ alone contributes almost to one third of the total $\chi^{2}$. This might indicate that $c_{P}$ receives important higher order contributions that are not being taken into account in the $O\left(p^{6}\right)$ calculation. Once again we obtain a crude estimate of the size of higher order ChPT corrections, by changing $f_{\pi}$ by $f_{0}$ in the last term of the expansion. $c_{P}$ suffers indeed the largest change, by almost $80 \%$.

Thus, we proceed to fit again all threshold parameters except $c_{P}$. The result is shown in the third row of Table 2. The fit quality improves sizably, but we still get a high $\chi^{2} /$ d.o.f. $=2.9$, which indicates that the two-loop calculation may not be enough to describe even the remaining threshold parameters with their current level of precision. Again, we see the effect of higher order corrections by making a fit replacing $f_{\pi}$ by $f_{0}$ in the $O\left(p^{6}\right)$ terms. We show the results in the fourth row of Table 2. Surprisingly, we now obtain a good $\chi^{2} /$ d.o.f. $=1.0$ and all LECs are less than two standard deviations away from those obtained by fitting only the threshold parameters with an $O\left(p^{4}\right)$ polynomial part. We conclude that, by excluding $c_{P}$, the two-loop fit can give an acceptable description of the rest of threshold parameters. For this reason, we have once more made a weighted average of the two fits (the one using $f_{\pi}$ and the one using $f_{0}$ ) adding systematic uncertainties to cover both sets. This we show in the fifth row of Table 2, where we can see that they are also quite compatible with previous determinations in the literature [5]. Also, this LECs satisfy again the axiomatic constraints [6].

\section{Summary}

We have reported a work [9] on the determination the low energy constants of $S U$ (2) Chiral Perturbation Theory (ChPT) at one and two loops by fitting to the threshold parameters obtained from sum rules using a recent and precise dispersive analysis of data [10], together with six additional observables that we have studied here.

We have checked that the one-loop formalism is clearly insufficient to accommodate the present level of precision. The $\chi^{2} /$ d.o.f. improves remarkably when using the two-loop expansion, although it is still not sufficient to get a good quality fit. This suggests that even higher order ChPT contributions may still be required to describe all these observables simultaneously. 
Determination of ChPT LECs from a precise description of $\pi \pi$ scattering threshold parameters Guillermo Rios

\begin{tabular}{lccccccc}
\hline \hline Fit to & $\bar{b}_{1}$ & $\bar{b}_{2}$ & $\bar{b}_{3}$ & $\bar{b}_{4}$ & $\bar{b}_{5}$ & $\bar{b}_{6}$ & $\frac{\chi^{2}}{\text { d.o.f. }}$ \\
\hline$a_{S}, b_{S}, a_{P}, a_{D}, c_{S}, b_{P}$ & $-14 \pm 4$ & $14.6 \pm 1.2$ & $-0.29 \pm 0.05$ & $0.76 \pm 0.02$ & $0.1 \pm 1.1$ & $2.2 \pm 0.2$ & 1.2 \\
All & $-2 \pm 3$ & $14.2 \pm 1.0$ & $-0.39 \pm 0.04$ & $0.746 \pm 0.013$ & $3.1 \pm 0.3$ & $2.58 \pm 0.12$ & 5.2 \\
All but $c_{P}$ & $-6 \pm 3$ & $15.9 \pm 1.0$ & $-0.36 \pm 0.04$ & $0.753 \pm 0.013$ & $2.2 \pm 0.4$ & $2.44 \pm 0.12$ & 2.9 \\
All but $c_{P}$, using $f_{0}$ & $-12 \pm 3$ & $13.9 \pm 0.9$ & $-0.30 \pm 0.04$ & $0.726 \pm 0.013$ & $1.0 \pm 0.3$ & $1.93 \pm 0.08$ & 1.04 \\
\hline Estimate $O\left(p^{6}\right)$ & $-10.5 \pm 5.1$ & $14.5 \pm 1.8$ & $-0.31 \pm 0.06$ & $0.73 \pm 0.02$ & $1.3 \pm 1.0$ & $2.1 \pm 0.4$ & - \\
\hline Ref. [5] & $-12.4 \pm 1.6$ & $11.8 \pm 0.6$ & $-0.33 \pm 0.07$ & $0.74 \pm 0.01$ & $3.6 \pm 0.4$ & $2.35 \pm 0.02$ & -
\end{tabular}

Table 2: $O\left(p^{6}\right)$ fits. In the first row we only fit to observables containing polynomial $O\left(p^{4}\right)$ contributions. Next we show the fit to all the threshold parameters obtained in this work. The quality is rather poor, but most of the disagreement is caused by $c_{P}$. When this observable is omitted, the resulting fits are much better, specially when using $f_{0}$ instead of $f_{\pi}$ in the last term of the ChPT expansion. We provide an estimate of the LECs uncertainties from the fits to all observables except $c_{P}$, as a weighted average of the fits using $f_{0}$ or $f_{\pi}$. The resulting $\bar{b}_{i}$ parameters are very consistent with previous determinations, listed in the last row.

\section{References}

[1] S. Weinberg, Physica A96 (1979) 327.

[2] J. Gasser and H. Leutwyler, Annals Phys. 158 (1984) 142;

[3] C. Riggenbach et al.,Phys. Rev. D 43, 127 (1991);

[4] G. Amoros, J. Bijnens and P. Talavera, Nucl. Phys. B 585, 293 (2000) [Erratum-ibid. B 598, 665 (2001)];

[5] G. Colangelo, J. Gasser and H. Leutwyler, Nucl. Phys. B 603, 125 (2001).

[6] B. Ananthanarayan, D. Toublan and G. Wanders, Phys. Rev. D 51, 1093 (1995); Z. -H. Guo, O. Zhang and H. Q. Zheng, AIP Conf. Proc. 1343, 259 (2011); A. V. Manohar and V. Mateu, Phys. Rev. D 77, 094019 (2008); V. Mateu, Phys. Rev. D 77, 094020 (2008); P. Dita, Phys. Rev. D 59, 094007 (1999);

[7] G. Colangelo et al., Eur. Phys. J. C 71, 1695 (2011).

[8] M. Knecht, B. Moussallam, J. Stern, N. H. Fuchs, Nucl. Phys. B457, 513-576 (1995).

[9] J. Nebreda, J. R. Pelaez and G. Rios, arXiv:1205.4129 [hep-ph].

[10] R. Garcia-Martin et al., R. Kaminski, J. R. Pelaez, J. Ruiz de Elvira, F. J. Yndurain, Phys. Rev. D83, 074004 (2011).

[11] G. Wanders, Helv. Phys. Acta 39, 228 (1966) M. G. Olsson, Phys. Rev. 162, 1338 (1967); F. P. Palou and F. J. Yndurain, Nuovo Cim. A 19, 245 (1974); J. L. Basdevant, J. C. Le Guillou and H. Navelet, Nuovo Cim. A 7, 363 (1972);B. Ananthanarayan, D. Toublan and G. Wanders, Phys. Rev. D 53, 2362 (1996); B. Ananthanarayan and P. Buettiker, Phys. Rev. D 54, 1125 (1996); Phys. Lett. B 415, 402 (1997); Eur. Phys. J. C 19, 517 (2001). G. Wanders, Helv. Phys. Acta 70, 287 (1997); G. Wanders, Phys. Rev. D 56, 4328 (1997); B. Ananthanarayan, G. Colangelo, J. Gasser and H. Leutwyler, Phys. Rept. 353, 207 (2001);

[12] J. R. Batley et al. [ NA48-2 Collaboration ], Eur. Phys. J. C70, 635-657 (2010).

[13] J. R. Pelaez and F. J. Yndurain, Phys. Rev. D 71, 074016 (2005); R. Kaminski, J. R. Pelaez and F. J. Yndurain, Phys. Rev. D 77, 054015 (2008)

[14] J. Bijnens, G. Colangelo, G. Ecker, J. Gasser, M. E. Sainio, Phys. Lett. B374, 210-216 (1996); Nucl. Phys. B508, 263-310 (1997) [Erratum-ibid. B 517, 639 (1998)]. 